\title{
THE ROLE OF LONG PERIODS OF INFECTIOUSNESS IN THE DYNAMICS OF ACQUIRED IMMUNODEFICIENCY SYNDROME (AIDS)
}

\begin{tabular}{ll}
\multicolumn{1}{c}{ By } \\
$\begin{array}{ll}\text { Carlos Castillo-Chavez } \\
\text { Cornell University } \\
\text { Ithaca, NY 14853 }\end{array}$ & $\begin{array}{l}\text { Kenneth Cooke } \\
\text { Pomona College } \\
\text { Claremont, CA } 91711\end{array}$ \\
$\begin{array}{l}\text { Wenzhang Huang } \\
\text { Claremont Graduate School } \\
\text { Claremont, CA 91711 }\end{array}$ & $\begin{array}{l}\text { Simon A. Levin } \\
\text { Cornell University } \\
\end{array}$ \\
& Ithaca, NY 14853 \\
$1-M$ & \\
\end{tabular}

$\mathrm{BU}-1011-\mathrm{M}$

Revised January 1989

In: Mathematical approaches to resource management and epidemiology. Lecture Notes in Biomathematics. (C. Castillo-Chavez, S.A. Levin and C. Shoemaker, eds.) Springer Verlag (in press). 

IMMUNODEFICIENCY SYNDROME (AIDS)

Carlos Castillo-Chavez Center for Applied Math. Ecology and Systematics Biometrics Unit Cornell University Ithaca, NY 14853-7801
Kenneth Cooke Dept. of Mathematics Pomona College Claremont, CA 91711
Wenzhang Huang Claremont Graduate School Claremont, CA 91711
Simon A. Levin Cent. for Environ. Research Ecol. and Syst. Cornell University Ithaca, NY 14853

Abstract.

Single and multiple group models for the spread of HIV (human immunodeficiency virus) are introduced. Partial analytical results for these models are presented for two specific cases. First for a model for which the duration of infectiousness has a negative exponential distribution and second for a model for which all individuals remain infectious for a fixed length of time.

1. Introduction

The discovery by Barre-Sinoussi's and Gallo's groups $[1,2,3,4,5]$ that HIV (human immunodeficiency virus) is the etiological agent for AIDS has brought an unprecedented amount of research on the biology of this retrovirus. At present, however, there is not enough understanding on the consequences of its transmission at the population level. Some routes of HIV transmission are through sex (direct, anal, and oral), through needle sharing, through blood transfusions and through vertical transmission (mother to child at birth). Important epidemiological factors involved in its transmission include: variable infectivity $[6,7,8]$, long periods of infectiousness [9] of eight years or more and cofactors (e.g. whether or not the HIV carrier is infected with another venereal disease). In addition, biological and sociodemographic factors such as sex, age, economic status, race, sexual preference, geographical area of residence, and the nature of the social networks that are particular to each culture, have to be taken into consideration if we are to understand the dynamics of HIV.

This epidemic already has raised many, some perhaps unsolvable, moral, practical, economical and ethical questions regarding the possible implementation of a variety of extreme intervention plans. These include random testing of the population, random testing of specific ethnic groups and the possibility of putting (known) infected individuals in quarantine (see [10] for a commentary). The testing of the possible effectiveness of (such extreme) intervention plans may only make sense in a realistic mathematical framework. However, any mathematical model has its faults. There is always a tradeoff between detail and tractability, and there are inherent limits to predictability. Although mathematical models can suggest possible consequences of intervention plans and assist in thinking about complex issues, we strongly feel that the numerical and mathematical results obtained through their use should not be used to circumvent the moral and ethical questions raised by this epidemic. 
In this paper, we report on a series of models that we have developed recently and that are extensions of those of Anderson et al. [11,12]. Our objective has been to identify the role played by the long period of infectiousness associated with HIV on the dynamics of sexually transmitted HIV in homogeneous and heterogeneous populations. We present only a brief description of these models and a partial list of our analytical results. Extensions and proofs of these results can be found in Castillo-Chavez et al. [13-14]. We note that some of our results partially overlap with some of the results obtained simultaneously and independently by Blythe and Anderson [15].

\section{Single group models}

In this section we describe two models with alternative distributions of the duration of infection. First we assume, as is commonly done in epidemiological models, that individuals are transfered out of the infected class at a constant rate, or equivalently that the duration of infection has a negative exponential distribution (Hethcote et al. [16]). For our second model, we assume that all infected individuals remain infectious for a fixed length of time. This approach allows us to compare the effect of the mean infectious period on the reproductive number (i.e. the number of secondary infections generated by a single infectious individual in a purely susceptible population) and therefore to understand better its role on the dynamics of HIV in a homogeneous population.

\section{a. Model with exponential removal}

We divide the population --sexually active male homosexuals with multiple partners-- into five groups: S (susceptibles) , I (infected that will develop "full-blown". AIDS), Y (infected that will not develop full-blown AIDS), $\mathbf{Z}$ (former $\boldsymbol{Y}$ individuals that are no longer sexually active), and $\mathbf{A}$ (former $\mathbf{I}$ individuals that have developed "full-blown" AIDS). Note that $\mathbf{A}$ and $\mathbf{Z}$ are cumulative classes and hence once individuals move into these classes they no longer enter into the dynamics of the disease; however, for bookkeeping purposes, we keep them on record. We do not include a latent class (i.e., those exposed individuals that are not yet infectious). Furthermore, we assume that once an individual develops full-blown AIDS or enters the $Z$ class, he is no longer infectious because he has no sexual contacts. We also assume that all infected individuals become immediately infectious, and that they stop being sexually active or acquire AIDS at the constant rates $\alpha_{\gamma}$ and $\alpha_{1}$; hence $1 / \alpha_{1}$ denotes the average infectious period and $1 / \alpha_{Y}$ the average sexual longevity of an individual. In addition, we let $\Lambda$ denote the recruitment rate into the susceptible class (defined to be those individuals who are homosexually active); $\mu$, the natural mortality rate; $\mathbf{d}$, the disease-induced mortality due to AIDS; $\mathbf{p}$, that fraction of the susceptibles that become infectious and will go into the 
AIDS class; and therefore (1-p), the fraction of susceptible individuals that become infectious and will not develop full blown AIDS. Following Anderson et. al. [11] and using

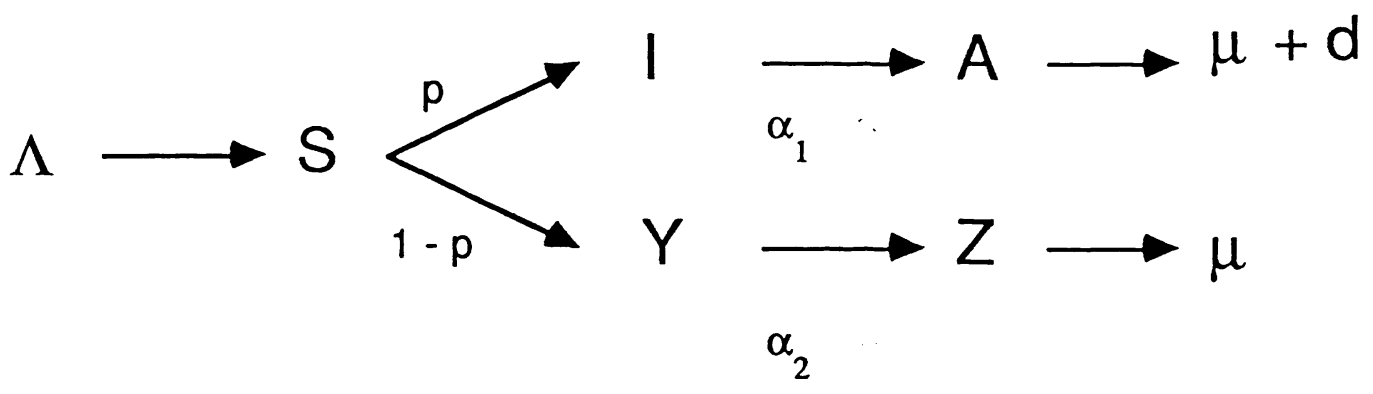

Fig. 1: Flow diagram for a single group model with exponential removal, for details see the text.

Figure 1, we arrive at the following simple epidemiological model with exponential removal:

$$
\begin{aligned}
& \frac{d S(t)}{d t}=\Lambda-\lambda C\left[\Pi(t) \frac{S(t) W(t)}{T(t)}-\mu S(t),\right. \\
& \frac{d l(t)}{d t}=\lambda p C\left[\Pi(t) \frac{S(t) W(t)}{T(t)}-\left(\alpha_{1}+\mu\right) I(t),\right. \\
& \frac{d Y(t)}{d t}=\lambda(1-p) C\left[\Pi(t) \frac{S(t) W(t)}{T(t)}-\left(\alpha_{Y}+\mu\right) Y(t),\right. \\
& \frac{d A(t)}{d t}=\alpha_{1} I(t)-(d+\mu) A(t), \\
& \frac{d Z(t)}{d t}=\alpha_{Y} Y(t)-\mu Z(t),
\end{aligned}
$$

where

$$
\mathrm{W}=\mathrm{I}+\mathrm{Y} \text { and } \mathrm{T}=\mathrm{W}+\mathrm{S} \text {. }
$$

Here the function $\mathrm{C}[T]$ denotes the mean number of sexual partners an average individual has per unit time, given that the population density is $T$, and $\lambda$ (a constant) denotes the average sexual risk per partner. More specifically (as in Hyman and Stanley [17]), $\lambda=\mathbf{i} \phi$ where $\mathbf{i}$ denotes the probability of infection per sexual contact (with an infected individual) and $\phi$ denotes the average number of contacts per sexual partner. Hence, $\lambda C[T]$ denotes the transmission rate per unit time per infected partner. The factor $W / T$ is the probability that a contact of a susceptible with a randomly selected individual will be with an infectious individual. Since individuals in classes $A$ and $Z$ are not sexually active, $\lambda C[T] S W / T$ denotes the number of newly infected individuals per unit time. $\mathrm{C}[T]$ is usually assumed to be approximately linear for small $T$ and to approach a saturation level for a large T. For AIDS, it may be that $C[T]$ should be taken as proportional to $T^{\delta}$ for $0<\delta \leq 1$ for small populations, but treated as a constant for large populations. This is because there is some evidence (Kingsley et al. [18]) that the probability of seroconversion (infection) increases with the number of infected sexual partners for those individuals that practice receptive anal sex. We use a 
general functional form for $\mathrm{C}[\mathrm{T}]$ that includes both of the above choices as special cases in order to determine how this assumption affects the conclusions. Anderson and May [12] have shown that in a homogeneous (one-group) model, $\mathrm{C}[\mathrm{T}]$ should not be the mean number of sexual partners per unit time, but rather should be larger because of the important role played by highly active individuals who are more likely to acquire infection and are also more likely to transmit it.

Unfortunately, there is evidence that AIDS is actually a progressive disease and that most individuals that have been infected will go on to develop "full-blown" AIDS. If we accept this view, then $p$ is approximately equal to one and equations (1.3) and (1.5) are no longer necessary. In the rest of this article we will report results only for the case $p=1$ : for the case $0<p<1$ the reader is referred to [13-14]. Observe that the dynamics of the classes $S$ and $I$ are governed autonomously, and hence the system (1.1), (1.2), (1.4) can be reduced to

$$
\begin{aligned}
& \frac{d S}{d t}=\Lambda-\lambda C[T] S \frac{l}{T}-\mu S, \\
& \frac{d l}{d t}=I\left(\lambda C[T] \frac{S}{T}-\sigma\right),
\end{aligned}
$$

where $T=S+1, \sigma=\mu+\alpha_{1}$, and where we assume that $C[T]$ is an increasing function of $T$.

The system (1.7)-(1.8) always has the infection-free state

$$
(\mathrm{S}, \mathrm{W})=\left(\frac{\Lambda}{\mu}, 0\right),
$$

as an equilibrium.

For this model, the reproductive number $\mathrm{R}$, i.e. the number of secondary infections produced by an infectious individual in a purely susceptible population, is given by

$$
\mathrm{R}=\lambda \mathrm{C}\left[\frac{\Lambda}{\mu}\right] \frac{1}{\sigma}
$$

where we observe that $1 / \sigma$ denotes the mean infectious period. We note that if $R>1$ there exists a unique endemic equilibrium given implicitly by the unique positive solution to the system:

$$
\begin{aligned}
& S=\frac{T}{C \Pi \frac{\sigma}{\lambda},} \\
& I=\left(\frac{\Lambda}{\mu}-S\right) \frac{\mu}{\sigma} .
\end{aligned}
$$

For these equilibria, we have established the following results: 
The system (1.7)-(1.8) has a unique (positive) endemic state if and only if the reproductive number exceeds unity $(R>1)$. The infectionfree state (1.9) is globally asymptotically stable (relative to solutions for which $S(0) \geq 0, W(0) \geq 0$ ) whenever the reproductive number $R$ is less than unity $(R<1)$ and it is unstable when $R>1$. In addition when $R$ crosses 1 (from below) there is a transcritical bifurcation with the endemic equilibrium becoming globally stable.

\section{b. Models with constant incubation period}

As previously, we assume that all individuals become immediately infectious; hence with this formulation the incubation period is again taken to be equal to the infectious period. For the 1infected it is assumed to be constant $(\omega)$ and for the $Y$-infected it is assumed to be a constant $\tau$ equal to the average length of their sex-life. Therefore all infected (assumed infectious) individuals remain a fixed length of time $(\omega)$ or $(\tau)$ in their corresponding classes (more general forms of the model allow $(\omega)$ and $(\tau)$ to be distributed [13-14]). $I_{0}(t)$ and $Y_{0}(t)$ denote those individuals that were in either class $I$ or $Y$ at time $t=0$, and are still infectious; $Z_{0}(t)$, those individuals that were in class $Z$ at time $t=0$, and are still alive; and $A_{0}(t)$, those individuals that had already developed full-blown AIDS at time $t=0$, and are still alive. We assume that $Z_{0}(t)$ and $A_{0}(t)$ vanish for large enough $t$, i.e., in mathematical terms that they have compact support. Since $\omega$ denotes the infectious period and $\tau$ the average sex-life of an individual in this population, we assume that $I_{0}(t)=Y_{0}(t)=0$ for $t>\max (\omega, \tau)$. The function $H(x)$ that appears in the following is the Heaviside function, defined as being equal to 1 if $x>0$ and zero otherwise. The rest of the parameters are defined as in Section 1. Using these conventions, and with the aid of Figure 2,

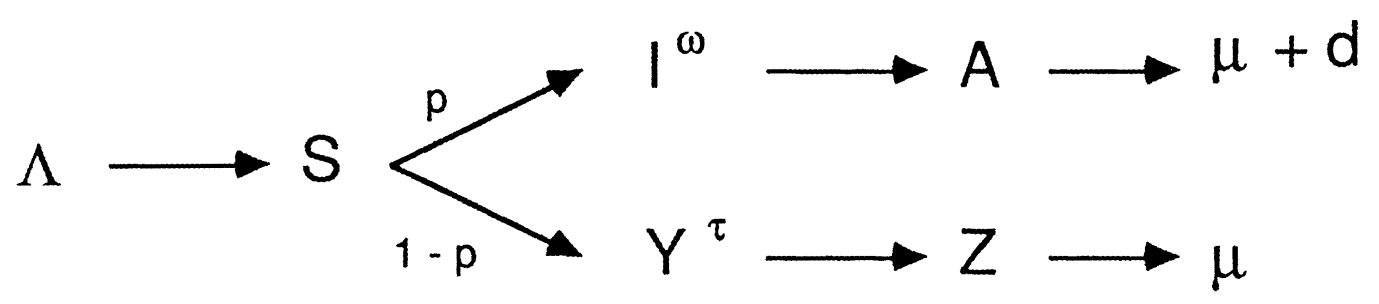

Fig. 2: Flow diagram for a single group model with constant periods of infectiousness, for details see the text.

we obtain the dynamical equations:

$$
\frac{d S(t)}{d t}=\Lambda-\lambda C[T](t) \frac{S(t) W(t)}{T(t)}-\mu S(t)
$$




$$
\begin{aligned}
& I(t)=I_{0}(t)+\lambda p \int_{t-\omega}^{t} C[T](x) \frac{S(x) W(x)}{T(x)} H(x) e^{-\mu(t-x)} d x \\
& Y(t)=Y_{0}(t)+\lambda(1-p) \int_{t-\tau}^{t} C\left[T(x) \frac{S(x) W(x)}{T(x)} H(x) e^{-\mu(t-x)} d x\right. \\
& A(t)=A_{0}(t)+\lambda p \int_{0}^{t-\omega} C[T](x) \frac{S(x) W(x)}{T(x)} H(x) e^{-\mu(t-x)-d(t-x-\omega)} d x \\
& Z(t)=Z_{0}(t)+\lambda(1-p) \int_{0}^{t-\tau} C[T](x) \frac{S(x) W(x)}{T(x)} H(x) e^{-\mu(t-x)} d x
\end{aligned}
$$

where $W(t)=I(t)+Y(t)$, and $T(t)=S(t)+W(t)$ and $C[T]$ is an increasing function of $T$. Observe that the classes $A$ and $Z$ are completely determined by the classes $S, Y$, and $I$. Hence we can restrict our analysis to the system given by (1.12)-(1.14). In addition, the results of Miller [19] and Londen [20] show that the initial population composition as expressed by, $I_{0}(t), Y_{0}(t), Z_{0}(t)$, and $A_{0}(t)$ will have a transient effect, but may be neglected for large enough $t$. The existence, uniqueness and positivity of solutions is established as follows:

First, we specify an appropriate set of initial conditions by setting $S(t)=r(t)$, $I(t)=p(t), Y(t)=m(t)$, on the interval $[-\max (\omega, \tau), 0]$. Moreover, in order to make this system consistent, we assume that $I_{0}(t)=p(t), Y_{0}(t)=m(t)$ on the interval $[-\max (\omega, \tau), 0]$. For this set of ordinary delay-differential equations local existence and uniqueness of solutions follows from standard results (see Hale, [21]).

We now assume that $I_{0}(t) \geq 0, Y_{0}(t) \geq 0$ on $[-\max (\omega, \tau), 0]$, and will show that the solutions remain nonnegative for $t>0$ as long as they are defined. That is, we will show that the point $(S(t), I(t), Y(t))$ remains in the nonnegative "orthant" in $\mathbf{R}^{3}$. To correspond to the biological context, we also assume that $S(t)>0$ on $[-\max (\omega, \tau), 0]$, that at least one of the infectious classes is strictly positive on a subinterval of $[-\max (\omega, \tau), 0]$, and that $\Lambda>0$.

The trajectory cannot reach a point in the face $S=0$, since if $S=0$ then $d S / d t$ $>0$ in a neighborhood by (1.12). Next we show that a solution cannot reach a face where either $I=0$ or $Y=0$. For, if $t^{*}$ is the first time that $I=0$ (or $Y=$ $0)$, then from (1.13)

$$
I(t) \geq \lambda p \int_{t^{*}-\omega}^{t^{*}} C[T](x) \frac{S(x) W(x)}{T(x)} H(x) e^{-\mu(t-x)} d x,
$$


which is a contradiction. Thus all variables are positive for $t>0$, under the stated conditions.

As before, we restrict our analysis to the case $p=1$ (that is we model AIDS as a progressive disease). In this case equation (1.14) is no longer relevant and the study of the steady states reduces to the following set of equations:

$$
\begin{aligned}
& \frac{d S(t)}{d t}=\Lambda-\lambda C[T] S(t) \frac{l(t)}{T(t)}-\mu S(t), \\
& \frac{d l(t)}{d t}=\lambda\left[C[T](t) S(t) \frac{I(t)}{T(t)}-C[T](t-\omega) S(t-\omega) \frac{l(t-\omega)}{T(t-\omega)} e^{-\mu \omega}\right]-\mu l(t),
\end{aligned}
$$

For this system the infection free-state $\left(\frac{\Lambda}{\mu}, 0\right)$ is always an equilibrium, in addition, the mean infectious period is given by $\frac{1-e^{-\mu \omega}}{\mu}$ and therefore, the reproductive number $R$, is given by

$$
R=\lambda C\left[\frac{\Lambda}{\mu}\right]\left(\frac{1-e^{-\mu \omega}}{\mu}\right)
$$

For this model we have established the following results

The system (1.17)-(1.18) has a unique positive endemic state if and only if the reproductive number exceeds unity $(R>1)$. The infectionfree state is globally asymptotically stable whenever the reproductive number $R$ is less than unity $(R<1)$ and it is unstable when $R>1$. In addition, the endemic state is locally asymptotically stable whenever $R>1$. Furthermore, periodic solutions do not arise when one varies parameters from either the endemic state or the infection-free state.

For extensions of these results to the case $0<p<1$ and to the case where distributed (rather than constant) delays are used the reader is referred to [13-14]. 
In this section we describe two multigroup models: the first assumes that individuals transfer from the infected class at a constant rate (i.e. that the duration of infection has a negative exponential distribution); in the second model we assume that all individuals remain infectious for a fixed length of time. In both models we assume that AIDS is a progressive disease; we relax this assumption in [13-14].

a. Model with exponential removal

A model with three subpopulations $(i=1,2,3)$ with different sexual and social practices is considered (in constructing this model, we follow the approach of Ross [22], and Hethcote and Yorke [23]). Group 1 includes those individuals whose sexual preferences, degree of sexual activity and social practices can facilitate the transmission of HIV. If we assume that the reservoir of the HIV virus is within the (sexually active) homosexual population, then Group 1 could include (sexually active) bisexuals, and perhaps a subgroup of the male and female population of prostitutes. Group 2 includes those heterosexual individuals who have multiple sexual partners, and Group 3 includes those essentially monogamous individuals whose risk of infection arises from social and sexual contact primarily with individuals of Group 2. This classification is somewhat arbitrary, but it is given primarily for the purpose of illustration. We denote by $\mathbf{S}_{\mathbf{i}}, \mathbf{I}_{\mathbf{i}}$, and $\mathbf{A}_{\mathbf{i}}$, the corresponding classes for group $\mathbf{i}$ as defined in Section 1. In this case $\mathbf{C}_{\mathbf{i}}[\mathrm{T}]=\mathbf{c}_{\mathbf{i}} \mathbf{C}[\mathrm{T}]$ ( $\mathrm{C}_{\mathbf{i}}$ appropriate constants for each group), $\lambda_{\mathbf{i}}, \Lambda_{\mathbf{i}}, \alpha$ and $\mu$ are defined as before but with a subindex to differentiate groups.

Proceeding as in Section 1 and guided by Figures 1 and 3 , we arrive at the following model:

$$
\begin{aligned}
& \frac{d S_{1}(t)}{d t}=\Lambda_{1}-\lambda_{1} c_{1} C[T](t) S_{1}(t) \frac{W(t)}{T(t)}-\mu S_{1}(t), \\
& \frac{d S_{2}(t)}{d t}=\Lambda_{2}-\lambda_{2} c_{2} C[T](t) S_{2}(t) \frac{W(t)}{T(t)}-\mu S_{2}(t), \\
& \frac{d S_{3}(t)}{d t}=\Lambda_{3}-\lambda_{3} c_{3} C[T](t) S_{3}(t) \frac{I_{2}(t)}{T(t)}-\mu S_{3}(t), \\
& \left.\frac{d l_{1}(t)}{d t}=\lambda_{1} c_{1} C_{1} T\right](t) S_{1}(t) \frac{W(t)}{T(t)}-\sigma l_{1}(t), \\
& \frac{d l_{2}(t)}{d t}=\lambda_{2} c_{2} C[T](t) S_{2}(t) \frac{W(t)}{T(t)}-\sigma I_{2}(t),
\end{aligned}
$$




$$
\frac{d l_{3}(t)}{d t}=\lambda_{3} c_{3} C[T](t) S_{3}(t) \frac{I_{2}(t)}{T(t)}-\sigma I_{3}(t),
$$

where $\sigma=\mu+\alpha$ and $T=I_{3}+S_{1}+S_{2}+S_{3}+W, W=I_{1}+I_{2}$.

Remark. Note that perhaps it would be more appropriate to use a different $T$ for the third group (i.e. $T_{2}=S_{2}+S_{3}+I_{2}+I_{3}$ ), and to use a constant instead of $c_{3} C[T]$. However, we feel that these changes would not affect the basic dynamics as they have a minimal effect in the reproductive number. Nevertheless, the use of a different $\mathrm{C}[\mathrm{T}]$ for each group may lead to complex dynamics. We are presently working on further elaborations of these models. For specific results on an n-group model that generalizes this model see Castillo-Chavez et al. [24].

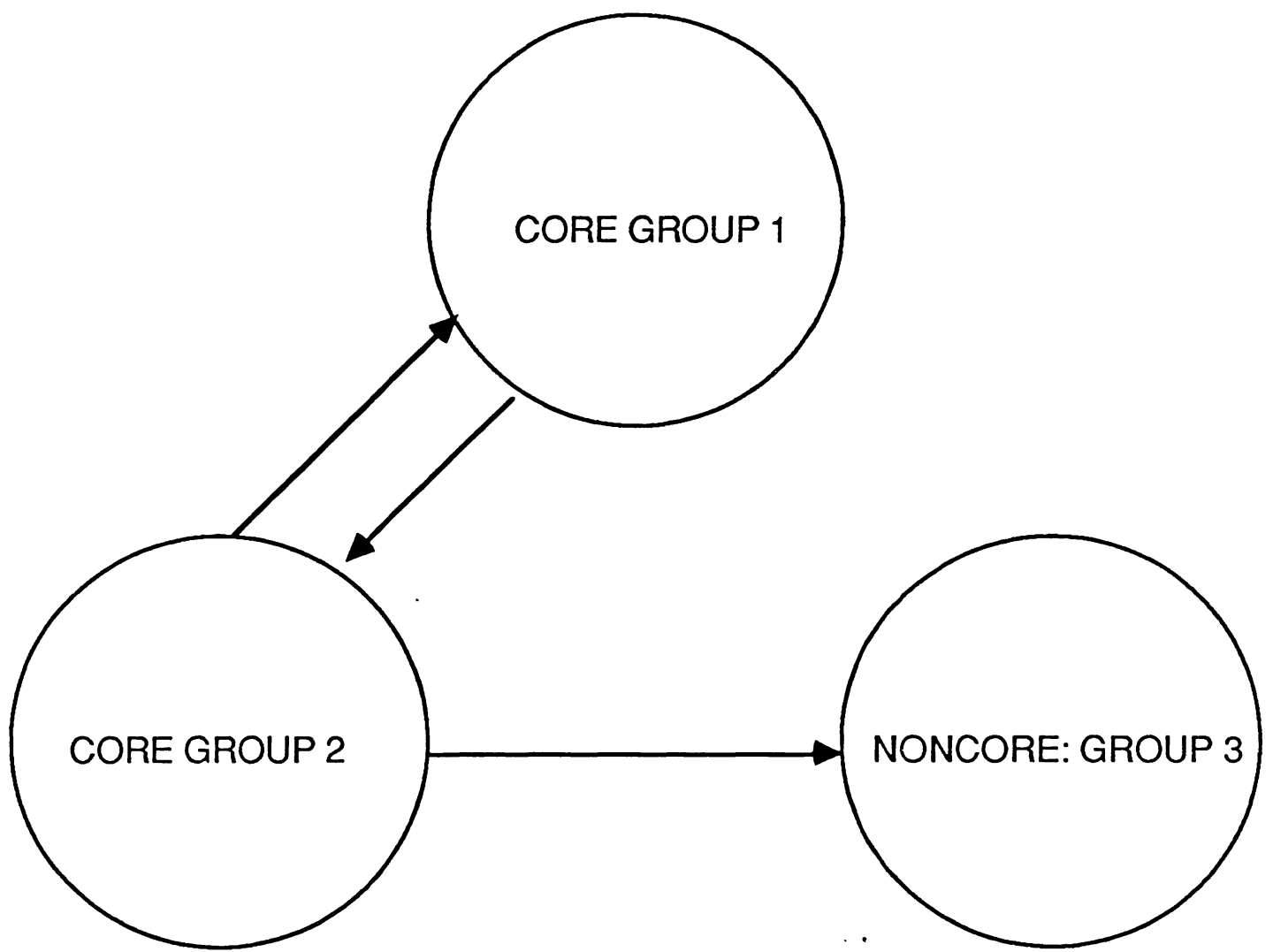

Fig. 3: Three group network, with two core groups and one noncore group, for details see the text.

This system always has the infection free-state

$$
\left(S_{1}, S_{2}, S_{3}, I_{1}, I_{2}, I_{3}\right)=\left(\frac{\Lambda_{1}}{\mu}, \frac{\Lambda_{2}}{\mu}, \frac{\Lambda_{3}}{\mu}, 0,0,0\right),
$$


as an equilibrium. In addition the reproductive number is given by the following expression:

$$
R=\frac{\Lambda_{1} \lambda_{1} c_{1}+\Lambda_{2} \lambda_{2} c_{2}}{\Lambda_{1}+\Lambda_{2}+\Lambda_{3}} c\left[\frac{\Lambda_{1}+\Lambda_{2}+\Lambda_{3}}{\mu}\right] \frac{1}{\sigma},
$$

which is the crucial parameter in the establishment of the following stability result.

\section{The infection-free state is locally asymptotically stable provided its reproductive number $R<1$, and is unstable if $R>1$.}

The stability analysis of endemic equilbria for a general $\mathrm{C}[\mathrm{T}]$ has not yet been fully resolved. In this case there may be more complicated dynamics. For further details on some partial results see [13-14].

b. Model with constant incubation period

If we now modify the previous model by assuming that I-infected individuals remain infected and infectious for a fixed length of time $(\omega)$, and ignore transient dynamics (as in Section lb) we then arrive at the following limiting model (using Figures 2 and 3):

$$
\begin{aligned}
& \frac{d S_{1}(t)}{d t}=\Lambda_{1}-\lambda_{1} c_{1} C[T](t) S_{1}(t) \frac{W(t)}{T(t)}-\mu S_{1}(t) \text {, } \\
& \frac{d S_{2}(t)}{d t}=\Lambda_{2}-\lambda_{2} c_{2} C[T](t) S_{2}(t) \frac{W(t)}{T(t)}-\mu S_{2}(t) \text {, } \\
& \frac{d S_{3}(t)}{d t}=\Lambda_{3}-\lambda_{3} c_{3} C[T](t) S_{3}(t) \frac{I_{2}(t)}{T(t)}-\mu S_{3}(t) \\
& \frac{d l_{1}(t)}{d t}=\lambda_{1} c_{1}\left[C[T](t) S_{1}(t) \frac{W(t)}{T(t)}-C[T](t-\omega) S_{1}(t-\omega) \frac{W(t-\omega)}{T(t-\omega)} e^{-\mu \omega}\right]-\mu l_{1}(t) \text {, } \\
& \frac{d l_{2}(t)}{d t}=\lambda_{2} c_{2}\left[C[T](t) S_{2}(t) \frac{W(t)}{T(t)}-C[T](t-\omega) S_{2}(t-\omega) \frac{W(t-\omega)}{T(t-\omega)} e^{-\mu \omega}\right]-\mu l_{2}(t), \\
& \frac{d l_{3}(t)}{d t}=\lambda_{3} c_{3}\left[C[T](t) S_{3}(t) \frac{I_{2}(t)}{T(t)}-C[T](t-\omega) S_{3}(t-\omega) \frac{I_{2}(t-\omega)}{T(t-\omega)} e^{-\mu \omega}\right]-\mu_{2}(t),
\end{aligned}
$$

where $T=I_{3}+S_{1}+S_{2}+S_{3}+W, W=I_{1}+I_{2}$.

This system always has the infection free-state 


$$
\left(\mathrm{S}_{1}, \mathrm{~S}_{2}, \mathrm{~S}_{3}, \mathrm{l}_{1}, \mathrm{I}_{2}, \mathrm{I}_{3}\right)=\left(\frac{\Lambda_{1}}{\mu}, \frac{\Lambda_{2}}{\mu}, \frac{\Lambda_{3}}{\mu}, 0,0,0\right),
$$

as an equilibrium. In addition the reproductive number is given by the following expression:

$$
\mathrm{R}=\frac{\Lambda_{1} \lambda_{1} c_{1}+\Lambda_{2} \lambda_{2} c_{2}}{\Lambda_{1}+\Lambda_{2}+\Lambda_{3}} \mathrm{C}\left[\frac{\Lambda_{1}+\Lambda_{2}+\Lambda_{3}}{\mu}\right]\left(\frac{1-e^{-\mu \omega}}{\mu}\right)
$$

which is the crucial parameter in the establishment of the following results:

The infection-free state is locally asymptotically stable provided its reproductive number $R<1$, and unstable if $R>1$. Furthermore, periodic solutions do not bifurcate from this state when parameters are varied.

The local stability analysis of endemic equilibria for a general $C[T]$ has not yet been fully resolved. For some partial results in this direction and for partial results for the case $0<p<1$ (i.e. when AIDS is not assumed to be a progresive disease), see [13-14, and 24].

\section{Acknowledgments}

This work has been partially supported by NSF grants DMS-8406472 to Simon A. Levin, and DMS-8603450 to Kenneth L. Cooke. Carlos Castillo-Chavez' research has been partially supported by The Center for Applied Mathematics, the Office of the Provost at Cornell University as well as by a Ford Foundation Postdoctoral Fellowship for Minorities. We thank them all.

\section{REFERENCES}

[1] Barré-Sinoussi, F., J. -C. Chermann, F. Rey, M. T. Nugeyre, S. Chamaret, J. Gruest, C. Dauguet, C. Axler-Blin, F. Brun-Vézinet, C. Rouzioux, W. Rozenbaum and L. Montagnier. Isolation of a T-lymphotropic retrovirus from a patient at risk for acquired immune deficiency syndrome (AIDS). Science 220: 868-70 (1983).

[2] Gallo, R.C., S. Z. Salahuddin, M. Popovic, G. M. Shearer, M. Kaplan, B. F. Haynes, T. J. Palker, R. Redfield, J. Oleske, B. Safai, G. White, P. Foster and P. D. Markhamet. Frequent detection and isolation of sytopathic retroviruses (HTLV-III) from patients with AIDS and at risk for AIDS. Science 224: 50C-503 (1984).

[3] Gallo, R. C. The first human retrovirus. Scientific American 255: 88-98 (1986).

[4] Gallo, R.C. The AIDS virus. Scientific American 256: 47-56 (1987). 
[5] Wong-Staal, F. and R. C. Gallo. Human T-lymphotropic retroviruses. Nature 317: 1985: 395-403.

[6] Francis, D.F., P. M. Feorino, J. R. Broderson, H. M. McClure, J. P. Getchell, C. R. McGrath, B. Swenson, J. S. McDougal, E. L. Palmer, A. K. Harrison, F. Barre-Sinoussi, J. -C. Chermann, L. Montagnier, J. W. Curran, C. D. Cabradilla and V. S. Kalyanaraman. Infection of chimpanzees with lymphadenopathy-associated virus. Lancet 2: 1276-77 (1984).

[7] Salahuddin, S.Z., J. E. Groopman, P.D. Markham, M. G. Sarngaharan, R. R. Redfield, M. F. McLane, M. Essex, A. Sliski and R. C. Gallo. HTLV-III in symptom-free seronegative persons. Lancet 2: 1418-20 (1984).

[8] Lange, J. M. A., D. A. Paul, H. G. Huisman, et. al. Persistent HIV antigenaemia and decline of HIV core antibodies associated with transition to AIDS. Brit. Med. J. 293, 1986: 145962.

[9] Medley, G. F., R. M. Anderson, D. R. Cox, and L. Billard. Incubation period of AIDS in patients infected via blood transfusions. Nature 328: 719-21(1987).

[10] Gerberding, J. L. and D. K. Henderson. Design of rational infection control policies for human immunodeficiency virus infection. J. Infectious Diseases. Vol. 156, No. 6 : 861 864 (1987).

[11] Anderson, R.M., R. M. May, G. F. Medley, and A. Johnson. A preliminary study of the transmission dynamics of the human immunodeficiency virus (HIV), the causative agent of AIDS. IMA J. Math. Med. Biol. 3:229-263 (1986).

[12] Anderson, R.M. and R. M. May. Transmission dynamics of HIV infection. Nature 326: 137142 (1987).

[13] Castillo-Chavez, C., Cooke, K., Huang, W. and Levin, S.A. On the role of long infectious periods in the dynamics of HIVIAIDS. Part 1. Single population models. J. of Math. Biol. (in press).

[14] Castillo-Chavez, C., Cooke, K., Huang, W. and Levin, S.A. The role of infectious periods in the dynamics of HIVIAIDS. Part 2. Multiple group models (in preparation).

[15] Blythe, S. P. and R. M. Anderson. Distributed incubation and infectious periods in models of the transmission dynamics of the human immunodeficiency virus (HIV). IMA J. Math. Med. Bio. (in press).

[16] Hethcote, H. W., H. W. Stech and P. van den Driessche. Periodicity and stability in epidemic models: a survey. In : Differential Equations and Applications in Ecology, Epidemics and Population_Problems. S. Busenberg and K. L. Cooke (eds.) Academic Press, New York: 65-82 (1981).

[17] Hyman, J. M. and E. A. Stanley. A risk based model for the spread of the AIDS virus. Math. Biosci. 90:415-473 (1988).

[18] Kingsley, R. A., R. Kaslow, C. R. Rinaldo Jr., K. Detre, N. Odaka, M. VanRaden, R. Detels, B. F. Polk, J. Chimel, S. F. Kersey, D. Ostrow and B. Visscher. Risk factors for seroconversion to human immunodeficiency virus among male homosexuals. The Lancet 1:345-348 (1987). 
[19] R. K. Miller. On the linearization of Volterra integral equations. J. Math. Anal. Appl. 23: 198208 (1968).

[20] S. O. Londen. Integral equations of Volterra type. In: Mathematics of Biology, M. lannelli (ed.), Liguori Editori, Napoli (1981).

[21] J. K. Hale. Theory of Functional Differential Equations. Springer Verlag. New York (1977).

[22] R. Ross. The Prevention of Malaria. 2nd. Edition, Murray, London (1911).

[23] Hethcote, H. W. and J. A. Yorke. Gonorrhea Transmission Dynamics and Control. Lecture Notes in Biomathematics No. 56, Springer VerlagHeidelberg (1984).

[24] Castillo-Chavez, C., K. Cooke, W. Huang, and S. A. Levin. Results on the dynamics for models for the sexual transmission of the human immuno-deficiency virus (submitted to Applied Mathematics Letters). 